\title{
Propagation of One-Dimensional Waves from a Source in Random Motion
}

\author{
J. K. Knowles \\ Division of Engineering and Applied Science, California \\ Institute of Technology, Pasadena, California 91109
}

\begin{abstract}
This paper is concerned with the transient problem of waves in an infinite one-dimensional medium owing to a monochromatic source whose position $X(t)$ is a stochastic function of time. Asymptotic results for the farfield are given for the general class of stationary source motions and for the case in which $X(t)$ is a Wiener process, corresponding to Brownian motion.
\end{abstract}

\section{INTRODUCTION}

$\mathbf{I}^{\mathrm{N}}$ $\mathrm{N}$ this paper, we are concerned with the analysis of one-dimensional waves from a moving monochromatic source whose position is a stochastic function of time. Our interest in this situation arose during an investigation of a more complicated question pertaining to the reflection and transmission of waves from a particle in Brownian motion.

The problem treated here consists in determining the simplest statistical properties of the solution of an initial-value problem for the wave equation with a nonhomogeneous term that involves the random motion of the source. There are several physical problems for which this is an appropriate mathematical model. A simple one that serves to fix ideas concerns a stretched string, initially at rest, which occupies the entire $x$ axis in its undeflected state. At time $t=0$, a concentrated transverse force is suddenly applied to the string. As time increases, the magnitude of this force oscillates sinusoidally with a given frequency $\omega$, while the position of force at time $t$ is $x=X(t)$, where $X(t)$ is a stochastic process.

In the deterministic problem for which $X(t)=v t$, where $v$ is a constant, the corresponding mathematical problem is elementary and its solution exhibits the well-known features of the Doppler effect. ${ }^{1}$ The situation under investigation here might thus be regarded as a generalization from this very simplest of source motions to the opposite extreme, in which the source

\footnotetext{
${ }^{1}$ See, for example, J. W. Strutt Lord Rayleigh, Theory of Sound (Dover Publications, Inc., New York, 1945), Vols. 1 and 2; C. A. Coulson, Waves (Oliver \& Boyd Ltd., Edinburgh, 1952); T. P. Gill, The Doppler Effect (Academic Press, Ltd., London, 1965).
}

motion is chaotic and may be treated as a realization of a stochastic process.

After formulating the problem in detail in the following Section, we examine in Sec. II the special class of source motions for which the random process $X(t)$ is strictly stationary of first order. ${ }^{2}$ Without further assumptions, we derive an explicit asymptotic representation of the mean value of the solution in the farfield. This takes the form of a sinusoidal wave whose frequency is that of the source, but whose amplitude and phase are determined by the (one-dimensional) characteristic function associated with $X(t)$. The structure of this asymptotic result further shows that the inverse problem of determining the (one-dimensional) distribution of an unknown stationary random source motion $X(t)$ from a knowledge of the farfield behavior of the mean disturbance has a simple, and unique, solution.

If $X(t)$ is a nonstationary stochastic process, the results can be quite different, as we illustrate in Sec. III. There, we study the disturbance due to a source in Brownian motion, corresponding to the case in which $X(t)$ is a Wiener process. As in the stationary case, the mean position of the source is constant-we take it to be $x=0$-but now the rms position is $\gamma \sqrt{ } t$, where $\gamma$ is the diffusion constant characteristic of the Wiener process. An asymptotic analysis again furnishes the farfield approximation to the mean disturbance. The result, however, no longer reduces to a simple sinusoidal signal but consists instead of a symmetrical pair of sine waves, traveling in opposite directions, whose ampli-

${ }^{2}$ For an elementary discussion of the probabilistic terms used here, see E. Parzen, Stochastic Processes (Holden-Day, Inc., San Francisco, Calif., 1962). 
tudes decay exponentially on either side of their peak values. These maximum values of amplitude occur at $x= \pm c t$, where $c$ is the "sonic speed" of the wave equation at hand. The frequency $\omega^{*}$ of these sinusoidal waves differs from the frequency $\omega$ of the source. It is given by

$$
\omega^{*}=\omega\left[2 / 1+\left(1+\frac{4 \gamma^{4}}{c^{4}} \omega^{2}\right)^{\frac{1}{3}}\right]^{\frac{1}{2}} .
$$

While there is indeed an extensive literature concerning problems of random waves and oscillations, much of it is relevant here only in a general way. An extensive survey of work pertaining to random vibrations has been prepared by Crandall. ${ }^{3}$ Stochastic aspects of wavepropagation problems have been primarily those associated with random media. ${ }^{4}$

\section{FORMULATION OF THE PROBLEM}

We seek a solution $u(x, t)$ of the differential equation

$$
u_{x x}-\left(1 / c^{2}\right) u_{t t}=P \cos \omega t \delta[x-X(t)],
$$

where $c, P$, and $\omega$ are constants, $\delta$ is the Dirac delta function, and subscripts $x$ and $t$ indicate partial derivatives. The initial conditions to accompany Eq. 1 are

$$
u(x, 0)=u_{t}(x, 0)=0 \text {. }
$$

We consider Eq. 1 for $t>0,-\infty<x<\infty$.

For essentially any $X(t)$, a formal solution of the foregoing initial value problem can be constructed with the aid of a Fourier transform in $x$. It is given by

$$
\begin{array}{r}
u(x, t)=-\frac{c P}{2 \pi} \int_{-\infty}^{\infty} d \xi \int_{0}^{t} d \tau \exp \{i \xi[X(\tau)-x]\} \\
\times \cos \omega \tau \frac{\sin \xi c(t-\tau)}{\xi} .
\end{array}
$$

By reversing the order of integration in Eq. 3 and evaluating the $\xi$ integral explicitly, we find the alternate representation

$$
u(x, t)=-\frac{c P}{2} \int_{0}^{t} H\left[t-\tau-\frac{|X(\tau)-x|}{c}\right] \cos \omega \tau d \tau,
$$

where $H$ denotes the Heaviside unit step function.

Because of its subsequent relevance, we wish to point out a qualitative feature of the solution that is easily deducible from the representation (Eq. 4). Suppose that $X(t)$ is a continuously differentiable function for which

$$
\left|X^{\prime}(t)\right|<c, \quad t>0,
$$

${ }^{3}$ S. H. Crandall, "Random Vibrations," in Applied Mechanics Surveys, N. Abramson et al., Eds. (Spartan Books, Washington, D. C., 1966), pp. 681-689.

${ }^{4}$ See Stochastic Processes in Mathematical Physics and Engineering (American Mathematical Society, Providence, R. I., 1964); esp. V. Twersky, "On Propagation in Random Media of Discrete Scatterers," pp. 84-116; J. B. Keller, "Stochastic Equations and Wave Propagation in Random Media," pp. 145-170; W. C. Hoffman, "Wave Propagation in a General Random Continuous medium," pp. 117-144; and the references cited in these articles. corresponding to permanently subsonic source motion. It is readily shown that Inequality 5 implies $t-\tau-|X(\tau)-x| / c \leqq 0$ for all values of $\tau$ between 0 and $t$, provided that $|x-X(0)| \geqq c t$. We conclude from Eq. 4 that at time $t$ the disturbance initiated in the string by the suddenly applied force at position $x=X(0)$ at time $t=0$ has not reached stations $x$ for which $|x-X(0)|>c t$. Thus, there are no "supersonic" precursors if the source motion is always subsonic; otherwise such precursors are, in general, present.

For purposes of later comparison, we record the form taken by Eq. 4 for the special deterministic problem of a fixed monochromatic source. Setting $X(t) \equiv 0$ in Eq. 4 and carrying out the integration, we get

$$
\begin{array}{r}
u(x, t)=-(c P / 2 \omega) H(t-|x| / c) \sin \omega(t-|x| / c), \\
{[X(t) \equiv 0] .}
\end{array}
$$

When $X(t)$ is a stochastic process, we compute the mean, or expected, value of $u(x, t)$ from Eq. 4 as

$$
\begin{gathered}
E[u(x, t)]=-\frac{c P}{2} \int_{0}^{t} \cos \omega \tau E\left\{H\left[t-\tau-\frac{|X(\tau)-x|}{c}\right]\right\} d \tau \\
=-\frac{c P}{2} \int_{0}^{t} d \tau \cos \omega \tau \\
\quad \times \int_{-\infty}^{\infty} H\left(t-\tau-\frac{|z-x|}{c}\right) d F(z ; \tau),
\end{gathered}
$$

or

$$
\begin{array}{r}
E[u(x, t)]=-\frac{c P}{2} \int_{0}^{t} \cos \omega \tau\{F[x+c(t-\tau) ; \tau] \\
-F[x-c(t-\tau) ; \tau]\} d \tau
\end{array}
$$

(see Ref. 5), where

$$
F(z ; t)=\operatorname{Prob}\{X(t) \leqq z\}
$$

is the (one-dimensional) distribution function of $X(t)$.

Although we restrict our attention in this paper to the calculation of the first moment $E[u(x, t)]$, it is possible in principle to obtain the mean square of $u$, and hence the variance of $u$, from Eq. 4. However, the fourfold integrals that are encountered make such a computation extremely involved.

\section{STATIONARY SOURCE MOTIONS}

We now assume that the random process $X(t)$ has mean zero and is strictly stationary of first order. This corresponds to the assumption that the mechanism responsible for the random fluctuation does not change in time. As a consequence of the strict stationarity, the distribution function $F(z ; t)$ of $X(t)$ is independent of

${ }^{5}$ For a discussion of Stieltjes integrals and their properties, see H. Cramer, Mathematical Methods of Statistics (Princeton University Press, Princeton, N. J., 1946), or T. M. Apostol, Mathematical Analysis (Addison-Wesley Publ. Co., Inc., Reading, Mass., 1957).

The Journal of the Acoustical Society of America 


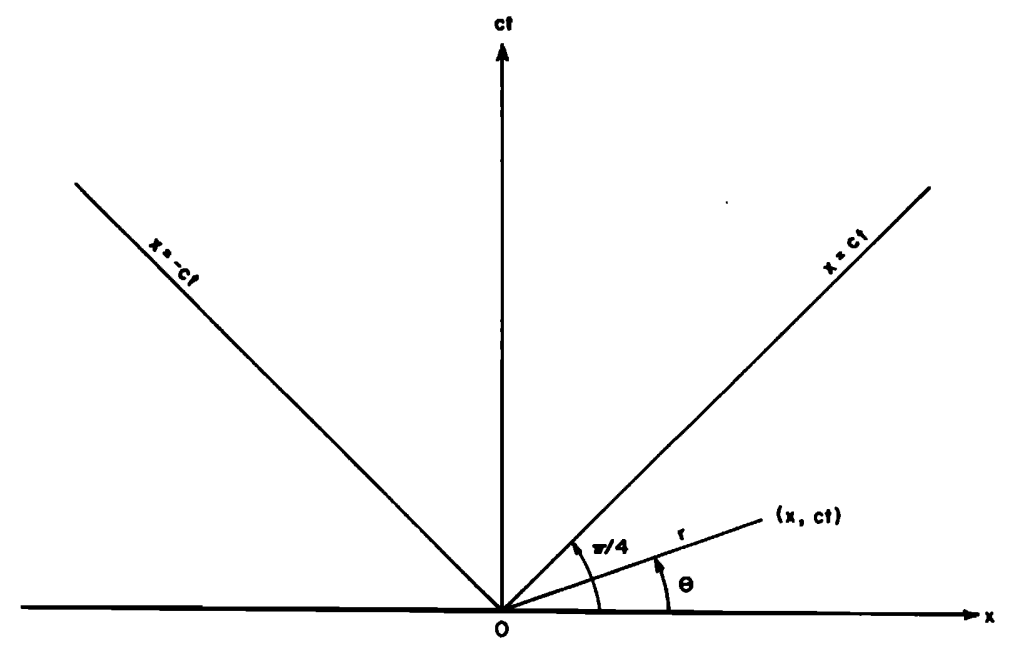

Fig. 1. $x$-ct plane.

$t$. We indicate this by writing

$$
F(z ; t)=F(z) \text {. }
$$

Finally, we assume that $X(t)$ has a finite second moment, so that

$$
\int_{-\infty}^{\infty} z^{2} d F(z)<\infty
$$

By writing Eq. 7 as the difference between two integrals and making suitable changes of variables, we obtain

$$
\begin{aligned}
E[u(x, t)]=-\frac{P}{2} & \left\{\int_{x}^{x+c t} \cos \omega\left(t+\frac{x-z}{c}\right) F(z) d z\right. \\
& \left.-\int_{x-c t}^{x} \cos \omega\left(t-\frac{x-z}{c}\right) F(z) d z\right\} .
\end{aligned}
$$

An integration by parts in each of the integrals in Eq. 11 yields

$$
\begin{array}{r}
E[u(x, t)]=-\frac{P c}{2 \omega}\left\{\int_{x}^{x+c t} \sin \omega\left(t+\frac{x-z}{c}\right) d F(z)\right. \\
\left.+\int_{x-c t}^{x} \sin \omega\left(t-\frac{x-z}{c}\right) d F(z)\right\},
\end{array}
$$

which in turn provides

$$
\begin{aligned}
E[u(x, t)]= & -\frac{P c}{2 \omega}\left\{\sin \omega\left(t+\frac{x}{c}\right) \int_{x}^{x+c t} \cos \frac{\omega z}{c} d F(z)\right. \\
& -\cos \omega\left(t+\frac{x}{c}\right) \int_{x}^{x+c t} \sin \frac{\omega z}{c} d F(z) \\
& -\sin \omega\left(t-\frac{x}{c}\right) \int_{x}^{x-c t} \cos \frac{\omega z}{c} d F(z) \\
& \left.-\cos \omega\left(t-\frac{x}{c}\right) \int_{x}^{x-c t} \frac{\omega z}{c} d F(z)\right\} .
\end{aligned}
$$

It is convenient to introduce functions $U_{+}$and $U_{-}$defined by

$$
\begin{array}{r}
U_{ \pm}(x, t)=-\frac{P c}{2 \omega}\left\{\mp \sin \omega(t \mp-) \int_{c}^{x} \cos \frac{\omega z}{c} d F(z)\right. \\
\left.-\cos \omega\left(\begin{array}{c}
t \mp- \\
c
\end{array}\right) \int_{x}^{x \mp c t} \sin \frac{\omega z}{c} d F(z)\right\},
\end{array}
$$

so that Eq. 13 can be written in the form

$$
E[u(x, t)]=U_{+}(x, t)+U_{-}(x, t) .
$$

We shall see that, in the far field, $U_{+}$and $U_{-}$represent waves traveling in the positive $x$ and negative $x$ directions, respectively.

Before turning to the asymptotic behavior of the mean disturbance in the farfield, we introduce some further notation. It is convenient to employ polar coordinates $r, \theta$ in the $x-c t$ plane (Fig. 1). These are defined through

$$
x=r \cos \theta, \quad c t=r \sin \theta ;
$$

we have $0 \leq \theta \leq \pi, r \geqq 0$. The subsonic region is the wedge $\pi / 4<\theta<3 \pi / 4$, while the wedges $0 \leqq \theta<\pi / 4$ and $3 \pi / 4<\theta \leqq \pi$ constitute the supersonic regions.

If $g(z)$ is a complex-valued function of the real variable $z$, we write

$$
g(z)=o_{F}(1) \quad \text { as } \quad z \rightarrow \infty,
$$

if there exist positive constants $M$ and $k$ such that

$$
|g(z)| \leqq M \int_{k z}^{\infty} d F\left(z^{\prime}\right)
$$

for all sufficiently large $z$. Similarly, $g(z)=o_{F}(1)$ as $z \rightarrow-\infty$ if, for all sufficiently large values of $-z$,

$$
|g(z)| \leqq M \int_{-\infty}^{k z} d F\left(z^{\prime}\right)
$$


for some positive constants $M$ and $k$. In view of In- in the subsonic region. Consider, for example, $U_{+}$from equality $10, g(z)=o_{F}(1)$ as $z \rightarrow \pm \infty$ implies that $g(z)=o\left(z^{-2}\right)$ as $z \rightarrow \pm \infty$, where the small $o$ without subscript has its usual meaning.

\section{A. Subsonic Farfield Approximation} Eq. 14,

$$
-\frac{2 \omega}{P c} U_{+}(x, t)=-\operatorname{Im} e^{i \omega(t-x / c)} \int_{x}^{x-c t} e^{i \omega z / c} d F(z),
$$

We begin by analyzing the asymptotic behavior of $U_{+}$and $U_{-}$, and hence of $E[u]$, as $r=\left(x^{2}+c^{2} t^{2}\right)^{\frac{1}{2}} \rightarrow \infty$

$$
\int_{x}^{x-c t} e^{i \omega z / c} d F(z)=-\int_{\sqrt{2} r \cos (\theta+\pi / 4)}^{x} e^{i \omega z / c} d F(z)=-\int_{-\infty}^{x} e^{i \omega z / c} d F(z)+\int_{-\infty}^{\sqrt{2} r \cos (\theta+\pi / 4)} e^{i \omega z / c} d F(z)
$$

If $\theta$ is fixed in the wedge $\pi / 4<\theta \leqq \pi$, we have $\cos (\theta+\pi / 4)<0$, so that

$$
\int_{x}^{x-c t} e^{i \omega z / c} d F(z)=-\int_{-\infty}^{x} e^{i \omega z / c} d F(z)+o_{F}(1), \text { as } \quad r \rightarrow \infty, \frac{\pi}{4}<\theta \leqq \pi .
$$

If $\theta$ is now further restricted to the interval $\pi / 4<\theta<\pi / 2$, we may write

$$
\int_{-\infty}^{x} e^{i \omega z / c} d F(z)=\int_{-\infty}^{\infty} e^{i \omega z / c} d F(z)-\int_{r \cos \theta}^{\infty} e^{i \omega z / c} d F(z)=\int_{-\infty}^{\infty} e^{i \omega z / c} d F(z)+o_{F}(1), \quad \text { as } \quad r \rightarrow \infty, \frac{\pi}{4}<\theta<\frac{\pi}{2} .
$$

Combining Eqs. 21 and 22, we obtain

$$
\int_{x}^{x-c t} e^{i \omega z / c} d F(z)=-\int_{-\infty}^{\infty} e^{i \omega z / c} d F(z)+o_{F}(1), \quad \text { as } \quad r \rightarrow \infty, \frac{\pi}{4}<\theta<\frac{\pi}{2} .
$$

On the other hand, if $\theta$ lies in the interval $\pi / 2<\theta \leqq \pi$, we have

$$
\int_{-\infty}^{x} e^{i \omega z / c} d F(z)=\int_{-\infty}^{r \cos \theta} e^{i \omega z / c} d F(z)=o_{F}(1), \quad \text { as } \quad r \rightarrow \infty, \frac{\pi}{2}<\theta \leqq \pi,
$$

which, when combined with Eq. 20, gives

$$
\int_{x}^{x-c t} e^{i \omega z / c} d F(z)=o_{F}(1), \text { as } \quad r \rightarrow \infty, \frac{\pi}{2}<\theta \leqq \pi .
$$

Using the estimates Eqs. 23 and 25 in Eq. 19, we find that

$$
U_{+}(x, t)=\left\{\begin{array}{c}
-\frac{P c}{2 \omega} \operatorname{Im}\left[e^{i \omega(t-x / c)} \int_{-\infty}^{\infty} e^{i \omega z / c} d F(z)\right]+o_{F}(1), \quad r \rightarrow \infty, \frac{\pi}{4}<\theta<\frac{\pi}{2}, \\
o_{F}(1), \quad r \rightarrow \infty, \quad \pi / 2<\theta \leqq \pi .
\end{array}\right.
$$

An analogous argument gives

$$
U_{-}(x, t)=\left\{\begin{array}{c}
o_{F}(1), \quad r \rightarrow \infty, \quad 0 \leqq \theta<\pi / 2, \\
-\frac{P C}{2 \omega} \operatorname{Im}\left[e^{i \omega(t+x / c)} \int_{-\infty}^{\infty} e^{i \omega z / c} d F(z)\right]+o_{F}(1), \quad r \rightarrow \infty, \quad \frac{\pi}{2}<\theta<\frac{3 \pi}{4} .
\end{array}\right.
$$

Combining Eqs. 26 and 27 in Eq. 15,

$$
E[u(x, t)]=-\frac{P c}{2 \omega} \operatorname{Im}\left[e^{i \omega(l-|x| / c)} \int_{-\infty}^{\infty} e^{i \omega z / c} d F(z)\right]+o_{F}(1), \quad \text { as } \quad r \rightarrow \infty, \quad \text { with } \quad \frac{\pi}{4}<\theta<\frac{\pi}{2} \text { or } \frac{\pi}{2}<\theta<\frac{3 \pi}{4} .
$$




\section{J . K. KNOWLES}

To obtain the subsonic farfield approximation (Eq. 28) in final form, we introduce the characteristic function $\Phi_{X}(\xi)$ of $X(t)$ :

$$
\Phi_{X}(\xi)=E\left(e^{i \xi X(t)}\right)=\int_{-\infty}^{\infty} e^{i \xi z} d F(z)
$$

Since $X(t)$ is a strictly stationary process, $\Phi_{X}(\xi)$ is independent of $t$. Equation 28 can be written as

$$
E[u(x, t)]=-\frac{P c}{2 \omega} \operatorname{Im}\left[e^{i \omega(t-|x| / c)} \Phi_{X}\left(\begin{array}{l}
\omega \\
c
\end{array}\right)\right]+o_{F}(1) .
$$

Writing

$$
\Phi_{X}(\xi)=\left|\Phi_{X}(\xi)\right| \exp \left[i \arg \Phi_{X}(\xi)\right],
$$

we obtain

$$
\begin{aligned}
E[u(x, t)]= & -\frac{P c}{2 \omega}\left|\Phi_{X}\left(\begin{array}{c}
\omega \\
c
\end{array}\right)\right| \\
& \times \sin \left[\omega\left(t-\frac{|x|}{c}\right)+\arg \Phi_{X}\left(\frac{\omega}{c}\right)\right] \\
& +o_{F}(1), \text { as } r \rightarrow \infty, \quad 0<|\theta-\pi / 2|<\pi / 4 .
\end{aligned}
$$

Equation 31 is our main result ${ }^{6}$ for stationary processes $X(t)$. It asserts that the subsonic farfield mean disturbance has the.form of a sine wave whose frequency $\omega$ is the same as that of the source and whose amplitude and phase are given in terms of the characteristic function of $X(t)$. We show below that nothing of importance propagates in the supersonic regions.

If we specialize to the deterministic case $X(t) \equiv 0$, the characteristic function $\Phi_{X}(\xi)$ is unity, and Eq. 31 reduces to Eq. 6 .

For a random source motion $X(t)$ whose distribution is symmetric with respect to the mean, $\Phi_{X}(\xi)$ is real and the phase of the asymptotic mean disturbance is independent of frequency.

Let

$$
\lambda=2 \pi c / \omega
$$

be the wavelength of the wave (Eq. 31). The amplitude in Eq. 31 is diminished from its value in the deterministic case $X(t) \equiv 0$, Eq. 6 , by a factor $|\Phi(2 \pi / \lambda)|$. Since

$$
\Phi_{X}(\xi)=1-\frac{1}{2} \sigma^{2} \xi^{2}+o\left(\xi^{2}\right), \quad \text { as } \quad \xi \rightarrow 0,
$$

where $\sigma^{2}$ is the variance of $X(t)$, the amplitude-reduction factor is given approximately by $1-\left(2 \pi^{2} \sigma^{2} / \lambda^{2}\right)$ for waves that are long in comparison with $\sigma$.

If the determination of the farfield approximation to the mean disturbance resulting from an oscillatory

\footnotetext{
6 Although Eq. 31 was derived for $\theta$ fixed in the open set $0<|\theta-\pi / 2|<\pi / 4$, it is easily verified by reviewing the argument in detail that Eq. 31 is valid uniformly in $\theta$ in the closed wedges $0<\epsilon \leqq|\theta-\pi / 4| \leqq \pi / 4-\epsilon$, for any positive $\epsilon$ less than $\pi / 4$.
}

source whose motion is a given stationary random process is regarded as the direct problem, we may refer to the inverse problem as that of finding the distribution of the unknown stationary random process $X(t)$, given the farfield amplitude and phase of the mean disturbance. Equation 31 shows that the inverse problem is solved immediately (and uniquely) once the farfield amplitude and phase are known over the entire frequency range $0 \leqq \omega<\infty$, since this information determines the modulus and argument of the characteristic function $\Phi_{X}(\xi)$, and $\Phi_{X}(\xi)$ in turn uniquely determines the (one-dimensional) distribution of $X(t)$ itself.

\section{B. Supersonic Farfield Approximation}

If no assumptions are made concerning the source motion beyond those of stationarity and the existence of the second moment, the sample functions $X(t)$ will not in general satisfy the condition (Eq. 5) for subsonic source motion. Indeed, the sample functions need not be differentiable unless additional assumptions are made. ${ }^{7}$ We must therefore expect that the general result (Eq. 13) for $E[u(x, t)]$ will exhibit supersonic precursors. For stationary processes, however, these are always small, as we show.

Let us first consider $U_{+}(x, t)$ as given by Eq. 19 . Setting $x=r \cos \theta$ in Eq. 21, we find that

$$
\int_{x}^{x-c t} e^{i \omega z / c} d F(z)=o_{F}(1), \text { as } r \rightarrow \infty, \frac{3 \pi}{4}<\theta \leqq \pi .
$$

In the remaining portion of the supersonic region, where $0 \leqq \theta<\pi / 4$, we write

$$
\int_{x}^{x-c t} e^{i \omega z / c} d F(z)=\int_{r \cos \theta}^{\sqrt{2} r \cos (\theta+\pi / 4)} e^{i \omega z / c} d F(z) .
$$

Since $\cos \theta$ and $\cos (\theta+\pi / 4)$ are both positive for $0 \leqq \theta<\pi / 4$, it follows that

$$
\begin{aligned}
\int_{x}^{x-c t} e^{i \omega z / c} d F(z)= & \int_{r \cos \theta}^{\infty} e^{i \omega z / c} d F(z) \\
& -\int_{\sqrt{2} r \cos (\theta+\pi / 4)}^{\infty} e^{i \omega z / c} d F(z) \\
= & o_{F}(1), \text { as } r \rightarrow \infty, 0 \leqq \theta<\pi / 4 .
\end{aligned}
$$

Combining Eqs. 33 and 35 and recalling Eq. 19, we find that in the supersonic regions $U_{+}$is given asymptotically by

$$
U_{+}(x, t)=o_{F}(1), \quad r \rightarrow \infty, \quad \pi / 4<|\theta-\pi / 2|<\pi / 2 .
$$

\footnotetext{
${ }^{7}$ Conditions that assure various degrees of smoothness of the sample functions of a stationary process are discussed in H. Cramér and M. R. Leadbetter, Stationary and Related Stochastic Processes (John Wiley \& Sons, Inc., New York, 1967).
} 
In an analogous way, it is found that

$U_{-}(x, t)=o_{F}(1), \quad$ as $\quad r \rightarrow \infty, \quad \pi / 4<|\theta-\pi / 2| \leqq \pi / 2$.

Equations 36, 37, and 15 show that the part of the mean disturbance that propagates at supersonic speed is asymptotically small. ${ }^{8}$

\section{Examples}

To illustrate the general Formula 31, we consider some special stationary stochastic processes $X(t)$.

\section{Stationary Normal Process}

If $X(t)$ is a stationary normal process with zero mean, its characteristic function is given by

$$
\Phi_{X}(\xi)=e^{-\frac{1}{2} \sigma^{2} \xi^{2}},
$$

where $\sigma^{2}$ is the variance of $X(t)$. In this case, Eq. 31 reduces to

$$
E[u(x, t)] \sim-\frac{P c}{2 \omega} \exp \left(-\frac{2 \pi^{2} \sigma^{2}}{\lambda^{2}}\right) \sin \omega\left(t-\frac{|x|}{c}\right),
$$

where the wavelength $\lambda$ is defined by Eq. 32. Only very long waves propagate with a substantial amplitude in this case.

\section{Sine Wave with Random Phase}

Consider an oscillatory source motion of the form

$$
X(t)=a \sin (\Omega t+\zeta)
$$

where the excursion $a$ and the frequency $\Omega$ are given constants, and $\zeta$ is a random variable with the uniform distribution on $[0,2 \pi]$. The stochastic process defined by Eq. 40 is strictly stationary of first order and its characteristic function is given by

$$
\Phi_{X}(\xi)=J_{0}(a \xi),
$$

where $J_{0}$ is the Bessel function of order zero.9 Equation 31 specializes to

$$
E[u(x, t)] \sim-\frac{P c}{2 \omega} J_{0}\left(\frac{2 \pi a}{\lambda}\right) \sin \omega\left(t-\frac{|x|}{c}\right) .
$$

It is interesting to note that the characteristic function, and hence the mean disturbance in the farfield, is independent of the frequency $\Omega$ of the source motion. In contrast to the result (Eq. 39) for stationary normal processes, the amplitude in Eq. 42 is an oscillatory function of the ratio of excursion $a$ to wavelength $\lambda$. In fact,

\footnotetext{
${ }^{8}$ It can be shown that Eq. 37 holds uniformly in $\theta$ for $\pi / 4+\epsilon$ $\leqq|\theta-\pi / 2| \leqq \pi / 2$, for any positive $\epsilon$ less than $\pi / 4$.

${ }^{9}$ See N. W. McLachlan, Bessel Functions for Engineers (Oxford University Press, London, 1941).
}

for the special wavelengths $\lambda_{k}$ given by $\lambda_{k}=2 \pi a / \alpha_{k}$, $k=1,2, \cdots$, where $\alpha_{k}$ is the $k$ th positive zero of $J_{0}(\alpha)$, the amplitude actually vanishes, showing that the mean disturbance is small in the farfield.

\section{Random Telegraph Signal ${ }^{2}$}

Cases in which the source motion is a discrete stationary random process are also included in the general result (Eq. 31). To illustrate, we suppose that $X(t)$ is of the form

$$
X(t)=X(0)(-1)^{N(t)}
$$

where $N(t)$ is a Poisson process, ${ }^{2}$ and the initial value $X(0)$ is either $a$ or $-a$ with equal probability. This corresponds to a source that jumps back and forth between the positions $x=a$ and $x=-a$, the times at which the jumps occur being determined by the Poisson process $N(t)$. The characteristic function of $X(t)$ is given $\mathrm{by}^{2}$

$$
\Phi_{X}(\xi)=\cos \xi a,
$$

and Eq. 31 accordingly specializes to

$$
E[u(x, t)] \sim-\frac{P c}{2 \omega} \cos \frac{2 \pi a}{\lambda} \sin \omega\left(t-\frac{|x|}{c}\right) .
$$

Again, the amplitude of the mean disturbance in the farfield vanishes at certain critical wavelengths.

\section{SOURCE IN BROWNIAN MOTION}

We turn now to the case in which the source motion $X(t)$ is a Wiener process. ${ }^{2}$ The distribution function associated with $X(t)$ is then given by

$F(z ; t)=\operatorname{Prob}\{X(t) \leq z\}=\frac{1}{(2 \pi)^{\frac{1}{2}} \gamma \sqrt{ } t} \int_{-\infty}^{z} \exp \left(-\frac{\zeta^{2}}{2 \gamma^{2} t}\right) d \zeta$,

where $\gamma^{2}$ is a diffusion constant characteristic of the Brownian motion.

We begin by substituting from Eq. 46 into the general formula of Eq. 7 to obtain the mean disturbance in the form

$$
\begin{aligned}
E[u(x, t)]=-\frac{c P}{2(2 \pi)^{\frac{1}{2}}} & \int_{0}^{t} d \tau \tau^{-\frac{1}{2}} \cos \omega \tau \\
& \times \int_{x-c(t-\tau)}^{x+c(t-\tau)} d \zeta \exp \left(-\frac{\zeta^{2}}{2 \gamma^{2} \tau}\right) .
\end{aligned}
$$

The change of variables $\zeta=\gamma \sqrt{ } \tau z$, followed by an inte- 
gration by parts with respect to $\tau$, leads to

$$
\begin{aligned}
E[u(x, t)]= & \frac{c P}{2(2 \pi)^{\frac{1}{2}} \omega} \int_{0}^{t} \sin \omega \tau \\
& \times \exp \left\{-\frac{[x+c(t-\tau)]^{2}}{2 \gamma^{2} \tau}\right\} \frac{\partial}{\partial \tau}\left[\frac{x+c(t-\tau)}{\gamma \sqrt{ } \tau}\right] d \tau \\
& -\frac{c P}{2(2 \pi)^{\frac{1}{2}} \omega} \int_{0}^{t} \sin \omega \tau \\
& \times \exp \left\{-\frac{[x-c(t-\tau)]^{2}}{2 \gamma^{2} \tau}\right\} \frac{\partial}{\partial \tau}\left[\frac{x-c(t-\tau)}{\gamma \sqrt{ } \tau}\right] d \tau .
\end{aligned}
$$

With the abbreviation

$$
\begin{aligned}
I(x, t)= & \frac{c P}{2(2 \pi)^{\frac{1}{\omega}} \omega} \int_{0}^{t} \sin \omega \tau \\
& \times \exp \left\{-\frac{[x+c(t-\tau)]^{2}}{2 \gamma^{2} \tau}\right\} \frac{\partial}{\partial \tau}\left[\frac{x+c(t-\tau)}{\gamma \sqrt{ } \tau}\right] d \tau,
\end{aligned}
$$

we may write Eq. 48 in the form

$$
E[u(x, t)]=I(x, t)+I(-x, t) .
$$

In Appendix A, we give the asymptotic analysis of $I(x, t)$ as $r=\left(x^{2}+c^{2} t^{2}\right)^{\frac{1}{2}} \rightarrow \infty$. This analysis shows that

$$
I(x, t)= \begin{cases}O\left(r^{-\frac{1}{2}} e^{-n r}\right) & \text { as } r \rightarrow \infty, \text { uniformly in } \theta \text { for } 0 \leqq \theta \leqq(\pi / 2)-\epsilon, \\ J[t+(x / c)]+O\left(r^{-\frac{1}{2}} e^{-n r}\right) & \text { as } r \rightarrow \infty, \text { uniformly in } \theta \text { for }(\pi / 2)+\epsilon \leqq \pi-\tan ^{-1 \frac{1}{2}} \\ O\left(r^{-\frac{1}{2}} e^{-n r}\right) & \text { as } r \rightarrow \infty, \text { uniformly in } \theta \text { for } \pi-\tan ^{-1 \frac{1}{2} \leqq} \leqq \pi\end{cases}
$$

where

$$
n=\min \left(\frac{2 c}{\sqrt{ } 5 \gamma^{2}}, \frac{c}{2 \gamma^{2}} \frac{\sin ^{2} \epsilon}{\cos \epsilon}\right),
$$

$\epsilon$ is any positive number less than $\pi / 4$, and

$$
\begin{aligned}
J\left(t+\frac{x}{c}\right)= & -\frac{c P}{4 \omega} \operatorname{sgn}\left(t+\frac{x}{c}\right) \operatorname{Im}\left[\left[1+\frac{\operatorname{sgn}\left(t+\frac{x}{c}\right)}{a}\right]\right. \\
& \left.\left.\times \exp \left\{-\frac{c^{2}}{\gamma^{2}}\left[a-\operatorname{sgn}\left(t+\frac{x}{c}\right)\right] \mid t+\frac{x}{c}\right)\right\}\right],
\end{aligned}
$$

where Im denotes imaginary part, sgnz denotes the sign of $z$, and

$$
\begin{aligned}
a=\left(1-\frac{2 i \gamma^{2} \omega}{c^{2}}\right)^{\frac{1}{2}}=\left[\frac{1}{2}+\frac{1}{2}(\right. & \left.\left.1+\frac{4 \gamma^{4} \omega^{2}}{c^{4}}\right)^{\frac{1}{2}}\right]^{\frac{1}{2}} \\
& -i\left[\frac{1}{2}\left(1+\frac{4 \gamma^{4} \omega^{2}}{c^{4}}\right)^{\frac{1}{2}}-1\right]^{\frac{1}{2}} .
\end{aligned}
$$

For brevity, we write Eq. 51 as

$$
I(x, t) \sim J(t+x / c) .
$$

From Eqs. 51 or 55, we infer that, in the farfield, $I(x, t)$ is asymptotically equivalent to a wave traveling in the direction of negative $x$, with a waveform given by Eq. 53. By Eq. 50, the mean disturbance in the farfield is then given asymptotically by the sum of two waves of identical waveform traveling in opposite directions:

$$
E[u(x, t)] \sim J(t+x / c)+J(t-x / c) .
$$

It is interesting to examine the waveform $J$ of Eq. 53 in detail. Consider first the nature of $J$ in the subsonic region $t+x / c>0$. In this range, Eq. 53 can be written as $J(t+x / c)=A_{1} e^{-\mu_{1}(t+x / c)} \sin \left[\omega^{*}(t+x / c)+\delta_{1}\right]$,

$$
t+x / c>0 \text {, }
$$

where the new frequency $\omega^{*}$ is given by

$\omega^{*}=\omega\left[2 / 1+\left(1+\frac{4 \gamma^{4}}{c^{4}} \omega^{2}\right)^{\frac{1}{3}}\right]^{\frac{1}{2}}$,

and

$$
\begin{aligned}
& A_{1}=-\frac{c P}{2 \omega}\left(\frac{\left[1+\left(1+\kappa^{2}\right)^{\frac{1}{2}}\right]^{\frac{1}{2}}\left\{\sqrt{2}+\left[1+\left(1+\kappa^{2}\right)^{\frac{1}{2}}\right]^{\frac{1}{2}}\right\}}{4\left(1+\kappa^{2}\right)^{\frac{1}{2}}}\right), \\
& \mu_{1}=\frac{c^{2}}{\gamma^{2}}\left(\left[\frac{1+\left(1+\kappa^{2}\right)^{\frac{1}{2}}}{2}\right]^{\frac{1}{2}}-1\right) \\
& \delta_{1}=\tan ^{-1}\left(\frac{\left[\left(1+\kappa^{2}\right)^{\frac{1}{2}}-1\right]^{\frac{1}{2}}}{\sqrt{2}\left(1+\kappa^{2}\right)^{\frac{1}{2}}+\left[\left(1+\kappa^{2}\right)^{\frac{1}{2}}+1\right]^{\frac{1}{3}}}\right)
\end{aligned}
$$

and we have introduced the notation

$$
\kappa=\left(2 \gamma^{2} / c^{2}\right) \omega .
$$

In the supersonic régime $t+x / c<0, J(t+x / c)$ is given by

$$
\begin{aligned}
J\left(t+\frac{x}{c}\right)=A_{2} e^{\mu_{2}(t+x / c)} \sin \left[\omega *\left(t+\frac{x}{c}\right)+\delta_{2}\right] & \\
& t+\frac{x}{c}<0,
\end{aligned}
$$

where the frequency $\omega^{*}$ is again given by Eq. 58 , and

$$
A_{2}=-\frac{c P}{2 \omega}\left(\frac{\left[1+\left(1+\kappa^{2}\right)^{\frac{1}{2}}\right]^{\frac{1}{3}}\left\{\left[1+\left(1+\kappa^{2}\right)^{\frac{1}{2}}\right]^{\frac{1}{2}}-\sqrt{2}\right\}}{4\left(1+\kappa^{2}\right)^{\frac{1}{2}}}\right)^{\frac{1}{3}}
$$




$$
\begin{aligned}
& \mu_{2}=\left(c^{2} / \gamma^{2}\right)\left\{\left[1+\left(1+\kappa^{2}\right)^{\frac{1}{2}} / 2\right]^{\frac{1}{3}}+1\right\}, \\
& \delta_{2}=\tan ^{-1} \frac{\left[\left(1+\kappa^{2}\right)^{\frac{1}{2}}-1\right]^{\frac{1}{2}}}{\sqrt{2}\left(1+\kappa^{2}\right)^{\frac{1}{2}}-\left[\left(1+\kappa^{2}\right)^{\frac{2}{2}}+1\right]^{\frac{1}{2}}},
\end{aligned}
$$

and $\kappa$ is again given by Eq. 62 .

Thus, the waveform $J(t+x / c)$ that describes the asymptotic structure of the leftward-traveling part of the mean disturbance represents an "exponentially amplitude-modulated" wave of frequency $\omega^{*}$. The intensity reaches a maximum at $x=-c t$ and decreases exponentially on either side of this peak. The rate of this exponential decay is greater in advance (i.e., on the supersonic side) of the peak, since $\mu_{2}>\mu_{1}$. The frequency $\omega^{*}$ of the sinusoidal part of the disturbance differs from the frequency of the source; from Eq. 58, it is found that $\omega^{*}$ is a monotone-increasing function of $\omega$. For low frequencies $\omega, \omega^{*} \sim \omega, \omega \rightarrow 0$, while for high frequencies $\omega^{*} \sim(c / \gamma) \sqrt{ } \omega, \omega \rightarrow \infty$.

It is easily verified that, when $\gamma \rightarrow 0$, Eq. 63 reduces to $J \equiv 0$ for $t+x / c<0$, while Eq. 57 becomes

$$
J\left(t+\frac{x}{c}\right)=-\frac{c P}{2 \omega} \sin \omega\left(t+\frac{x}{c}\right), \quad t+\underset{c}{x}>0 .
$$

This is to be expected, since the limit $\gamma \rightarrow 0$ should lead to the deterministic result (Eq. 6).

The presence of the supersonic precursor (Eq. 63) is, of course, a consequence of the failure of the condition of Eq. 5. Indeed, with probability 1, the sample functions $X(t)$ for the Wiener process are nowhere differentiable.

\section{Appendix A. Asymptotic Behavior of $I(x, t)$}

Here, we establish the asymptotic result Eqs. 51, 53 concerning the integral

$$
\begin{aligned}
I(x, t)= & \frac{c P}{2(2 \pi)^{\frac{1}{2}} \omega} \int_{0}^{t} \sin \omega \tau \\
& \times \exp \left\{-\frac{[x+c(t-\tau)]^{2}}{2 \gamma^{2} \tau}\right\} \frac{\partial}{\partial \tau}\left[\frac{x+c(t-\tau)}{\gamma \sqrt{ } \tau}\right] d \tau .
\end{aligned}
$$

If $t+x / c \neq 0$, we make the change of integration variable

$$
\tau=|t+x / c| / v^{2}
$$

in Eq. A1, obtaining, after considerable algebra, the formula

$$
\begin{aligned}
I(x, t)= & -\frac{c^{2} P}{2 \gamma \omega(2 \pi)^{\frac{1}{2}}} \operatorname{sgn}\left(t+\frac{x}{c}\right)\left(\left|t+\frac{x}{c}\right|\right)^{\frac{1}{2}} \\
& \times \operatorname{Im} \int_{|1+x / c t|^{\frac{1}{2}}}^{\infty}\left[1+\frac{\operatorname{sgn}\left(t+\frac{x}{c}\right)}{v^{2}}\right] . \\
& \times \exp \left[-\frac{c^{2}}{2 \gamma^{2}}\left|t+\frac{x}{c}\right|\left\{\left[\begin{array}{c}
\operatorname{sgn}\left(t+\frac{x}{c}\right) \\
v-\frac{i \kappa}{v}
\end{array}\right]-\frac{i}{v^{2}}\right]\right] d v,
\end{aligned}
$$

where Im denotes imaginary part, $\operatorname{sgn}(t+x / c)$ is +1 if $t+x / c>0,-1$ if $t+x / c<0$, and $\kappa$ is given by Eq. 62 .

\section{CASE 1. $|1+x / c t|>1$}

In the $x-c t$ plane of Fig. A-1, this case corresponds to those points for which $0 \leqq \theta<\pi / 2, r>0$, or for which $\pi-\tan ^{-1}\left(\frac{1}{2}\right)<\theta \leqq \pi, r>0$. Throughout the interval of integration in $\mathrm{Eq}$. $\mathrm{A} 3$, we have $v>1$. Thus

$$
\begin{aligned}
& |I(x, t)| \leqq \frac{c^{2} P}{2 \gamma \omega(2 \pi)^{\frac{1}{3}}}\left|t+\frac{x}{c}\right|^{\frac{1}{2}} \int_{|1+x / c t|^{\frac{1}{2}}}^{\infty}\left[1+\frac{\operatorname{sgn}\left(t+\frac{x}{c}\right)}{v^{2}}\right] \\
& \exp \left\{-\frac{c^{2}\left|t+\frac{x}{c}\right|}{2 \gamma^{2}}\left[v-\frac{\operatorname{sgn}\left(t+\frac{x}{c}\right)}{v}\right]^{2}\right\} d v .
\end{aligned}
$$

Setting $y=v-[\operatorname{sgn}(t+x / c)] / v$ in Eq. A4, we obtain

$$
\begin{aligned}
|I(x, t)| \leqq & \frac{c^{2} P}{2 \gamma \omega(2 \pi)^{\frac{1}{2}}}\left|t+\frac{x}{c}\right|^{\frac{1}{2}} \\
& \times \int_{v_{0}-\left[\operatorname{sgn}(t+x / c) / v_{0}\right]}^{\infty} \exp \left[-\frac{c^{2}\left|t+\frac{x}{c}\right|}{2 \gamma^{2}} y^{2}\right] d y,
\end{aligned}
$$

where

$$
v_{0}=|1+x / c t|^{\frac{3}{3}} .
$$

A change of scale in the integral Eq. A5 yields

$$
|I(x, t)| \leqq \frac{c P}{2 \omega} \frac{1}{(2 \pi)^{\frac{1}{2}}} \int_{w_{0}}^{\infty} e^{-\frac{1}{2} w 2} d w,
$$

where

$$
w_{0}=\frac{c}{\gamma}\left|t+\frac{x}{c}\right|^{\frac{1}{2}}\left\{v_{0}-\left[\operatorname{sgn}\left(t+\frac{x}{c}\right) / v_{0}\right]\right\}=\frac{x}{\gamma \sqrt{ } t} \operatorname{sgn}\left(t+\frac{x}{c}\right) .
$$




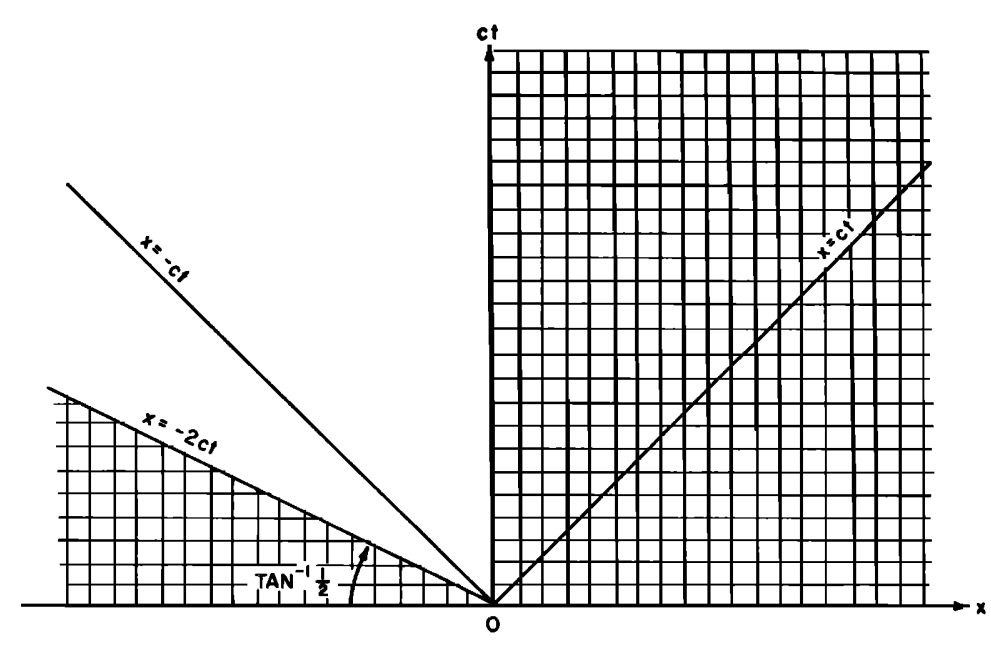

FIG. A-1. $x$-ct plane. Shaded area: Case 1, $|1+x / c t|>1$. Unshaded area: Case $2,|1+x / c t|<1$.

When $|1+x / c t|>1$, we have $x \operatorname{sgn}(t+x / c)=|x|$, so

$$
w_{0}=\frac{|x|}{\gamma \sqrt{ } t}=\frac{\sqrt{ } c}{\gamma} \sqrt{ } r\left[|\cos \theta| /(\sin \theta)^{\frac{1}{2}}\right],
$$

using the polar coordinates of Eq. 16. In the closed wedge $0 \leqq \theta \leqq(\pi / 2)-\epsilon$,

$$
w_{0} \geqq k(\epsilon) \sqrt{ } r,
$$

where $k(\epsilon)=(\sqrt{ } c / \gamma)\left[\sin \epsilon /(\cos \epsilon)^{\frac{1}{2}}\right]$. Here $\epsilon$ is positive and small. In the closed wedge $\pi-\tan ^{-1}\left(\frac{1}{2}\right) \leqq \theta \leqq \pi$,

$$
w_{0} \geqq\left(2 / \sqrt{ } 5^{\frac{1}{2}}\right)(\sqrt{ } c / \gamma) \sqrt{ } r .
$$

From Eqs. A7, A10, and A11, we obtain the estimates

$$
|I(x, t)| \leqq \begin{cases}\frac{c P}{2 \omega} \frac{1}{(2 \pi)^{\frac{1}{2}}} \int_{k(\epsilon) \vee r} e^{-\frac{1}{2} w^{2}} d w, & 0 \leqq \theta \leqq \frac{\pi}{2}-\epsilon, \\ \frac{c P}{2 \omega} \frac{1}{(2 \pi)^{\frac{1}{2}}} \int_{\left(2 / \mathcal{V}^{5}\right)(\sqrt{ } c / \gamma) \vee r}^{\infty} e^{-\frac{1}{2} w^{2} d w} \\ \pi-\tan ^{-1}\left(\frac{1}{2}\right) \leqq \theta \leqq \pi .\end{cases}
$$

of the integrals in A12, we obtain ${ }^{\mathrm{A} 1}$

$$
|I(x, t)| \leqq\left\{\begin{array}{l}
\frac{c P}{2 \omega} \frac{1}{(2 \pi)^{\frac{1}{2}}} \frac{1}{k(\epsilon) \sqrt{ } r} e^{-\frac{1}{2} k^{2}(\epsilon) r}, \\
\text { as } r \rightarrow \infty, \quad 0 \leqq \theta \leqq \frac{\pi}{2}-\epsilon, \\
\frac{c P}{2 \omega} \frac{1}{(2 \pi)^{\frac{1}{2}}} \frac{\sqrt{ } 5 \gamma}{2 \sqrt{ } c \sqrt{ } r} e^{-2 c r / \sqrt{ } 5 \gamma 2}, \\
\text { as } r \rightarrow \infty, \quad \pi-\tan ^{-1}\left(\frac{1}{2}\right) \leqq \theta \leqq \pi .
\end{array}\right.
$$

Thus, in the two wedges of the $x-c t$ plane corresponding to Case 1, the integrand is small in the farfield. In fact, as $r \rightarrow \infty$,

$$
\begin{aligned}
& I(x, t)=O\left(r^{-\frac{1}{2}} e^{-n r}\right) \quad \text { uniformly in } \theta \text { for } \\
& 0 \leqq \theta \leqq \frac{\pi}{2}-\epsilon \text { or } \quad \pi-\tan ^{-1}\left(\frac{1}{2}\right) \leqq \theta \leqq \pi,
\end{aligned}
$$

where

$$
\begin{aligned}
& n=\min \left(\frac{1}{2} k^{2}(\epsilon), 2 c / \sqrt{ } 5 \gamma^{2}\right) . \\
& \text { II. CASE 2. }|1+x / c l|<1
\end{aligned}
$$

We now consider the case $|1+x / c t|<1$, corresponding to the wedge $\pi / 2<\theta<\pi-\tan ^{-1}\left(\frac{1}{2}\right)$, and we write

where

$$
I(x, t)=J\left(t+\frac{x}{c}\right)-K(x, t)
$$

$$
\begin{aligned}
J\left(t+\frac{x}{c}\right)=-\frac{c^{2} P}{2 \gamma \omega(2 \pi)^{\frac{1}{2}}} \operatorname{sgn}\left(t+\frac{x}{c}\right)\left|t+\frac{x}{c}\right|^{\frac{1}{2}} \operatorname{Im} \int_{0}^{\infty}\{1+ & {\left.\left[\operatorname{sgn}\left(t+\frac{x}{c}\right) / v^{2}\right]\right\} } \\
& \times \exp \left[-\frac{c^{2}}{2 \gamma^{2}}\left|t+\frac{x}{c}\right|\left(\left\{v-\left[\operatorname{sgn}\left(t+\frac{x}{c}\right) / v\right]\right\}^{2}-\frac{i_{K}}{v^{2}}\right)\right] d v
\end{aligned}
$$

A1 W. Feller, An Introduction to Probability Theory and Its Applications (John Wiley \& Sons, New York, 1957), Vol. 1, p. 166. 
and

$$
\begin{aligned}
K(x, l)=-\frac{c^{2} P}{2 \gamma \omega(2 \pi)^{\frac{1}{2}}} \operatorname{sgn}\left(t+\frac{x}{c}\right)\left|l+\frac{x}{c}\right|^{\frac{1}{2}} \operatorname{Im} \int_{0}^{|1+x / c t| \frac{1}{2}} & \left\{1+\left[\operatorname{sgn}\left(t+\frac{x}{c}\right) / v^{2}\right]\right\} \\
& \times \exp \left[-\frac{c^{2}}{2 \gamma^{2}}\left|t+\frac{x}{c}\right|\left(\left\{v-\left[\operatorname{sgn}\left(t+\frac{x}{c}\right) / v\right]\right\}^{2}-\frac{i \kappa}{v^{2}}\right)\right] d v .
\end{aligned}
$$

An analysis similar to that carried out for Case 1 shows that, as $r \rightarrow \infty$,

$$
K(x, t)=O\left(r^{-\frac{1}{2}} e^{-n r}\right)
$$

uniformly in $\theta$ for

$$
\frac{\pi}{2}+\epsilon \leqq \theta \leqq \pi-\tan ^{-1}\left(\frac{1}{2}\right),
$$

where $m$ is again given by Eq. A15. Equations A16 and A19 show that $I(x, t)$ differs from $J(t+x / c)$ by small quantities in the farfield in Case 2. It remains only to compute $J$ explicitly.

\section{EVALUATION OF $J$}

According to Eq. A17,

where

$$
J(\xi)=-\frac{c^{2} P}{2 \gamma \omega(2 \pi)^{\frac{1}{2}}}(\operatorname{sgn} \xi)\left|t+\frac{x}{c}\right|^{\frac{1}{2}} \operatorname{Im} \hat{J}(\xi),
$$

$\hat{J}(\xi)=\int_{0}^{\infty}\left(1+\frac{\operatorname{sgn} \xi}{v^{2}}\right)$

Clearly,

$$
\times \exp \left\{-\frac{c^{2}}{2 \gamma^{2}}|\xi|\left[\left(v-\frac{\operatorname{sgn} \xi}{v}\right)^{2}-\frac{i \kappa}{v^{2}}\right]\right\} d v .
$$

$\hat{J}(\xi)=e^{\left(c^{2} / \gamma^{2}\right) \xi} \int_{0}^{\infty}\left(1+\frac{\operatorname{sgn} \xi}{v^{2}}\right)$

$$
\times \exp \left[-\frac{c^{2}|\xi|}{2 \gamma^{2}}\left(v^{2}+\frac{1-i \kappa}{v^{2}}\right)\right] d v \text {. }
$$

Let

$a=(1-i \kappa)^{\frac{1}{2}}=(1 / \sqrt{2})\left[1+\left(1+\kappa^{2}\right)^{\frac{1}{2}}\right]^{\frac{1}{2}}$

$$
-(i / \sqrt{2})\left[\left(1+\kappa^{2}\right)^{\frac{1}{2}}-1\right]^{\frac{1}{2}}
$$

and let $\sqrt{ } a$ be that square root of $a$ that lies in the fourth quadrant. Making the change of variables $v=\sqrt{ } a y$ in Eq. A22 leads to

$$
\begin{aligned}
\hat{J}(\xi)=\sqrt{ } a e^{c^{2} \xi / \gamma^{2}} \int_{\Gamma} & \left(1+\frac{\operatorname{sgn} \xi}{a y^{2}}\right) \\
& \times \exp \left[-\frac{c^{2} a|\xi|}{2 \gamma^{2}}\left(y^{2}+\frac{1}{y^{2}}\right)\right] d y,
\end{aligned}
$$

where the path $\Gamma$ is a straight line lying in the sector $0 \leqq \arg y<\pi / 4$ in the $y$ plane and connecting $y=0$ to $y=\infty$. It is easy to justify the deformation of contour that replaces $\Gamma$ by the positive real $y$ axis in Eq. A24, so that

$$
\begin{array}{r}
\frac{1}{\sqrt{ } a} e^{-c^{2} \xi / \gamma^{2}} \hat{J}(\xi)=\int_{0}^{\infty} \exp \left[-\frac{c^{2} a|\xi|}{2 \gamma^{2}}\left(y^{2}+\frac{1}{y^{2}}\right)\right] d y \\
+\frac{\operatorname{sgn} \xi}{a} \int_{0}^{\infty} \frac{1}{y^{2}} \exp \left[-\frac{c^{2} a|\xi|}{2 \gamma^{2}}\left(y^{2}+\frac{1}{y^{2}}\right)\right] d y .
\end{array}
$$

Replacing $y$ by $y^{-1}$ in the second integral in Eq. A25 shows that

$$
\begin{aligned}
\frac{1}{\sqrt{ } a} e^{-c^{2} \xi / \gamma^{2}} \hat{J}(\xi) & =\left(1+\frac{\operatorname{sgn} \xi}{a}\right) \\
& \times \int_{0}^{\infty} \exp \left[-\frac{a c^{2}|\xi|}{2 \gamma^{2}}\left(y^{2}+\frac{1}{y^{2}}\right)\right] d y .
\end{aligned}
$$

The integral in Eq. A26 is known ${ }^{\mathrm{A} 2}$; when it is evaluated, we find that

$$
\hat{J}(\xi)=\sqrt{\frac{\pi}{2}}_{\frac{\gamma}{c}}\left(1+\frac{\operatorname{sgn} \xi}{a}\right)|\xi|^{-\frac{1}{2}} \exp \left[-\frac{c^{2}}{\gamma^{2}}(a-\operatorname{sgn} \xi)|\xi|\right] .
$$

Equations A27 and A20 furnish

$$
\begin{aligned}
J(\xi)=-\frac{c P}{4 \omega} \operatorname{sgn} \xi \operatorname{Im} & \left\{\left(1+\frac{\operatorname{sgn} \xi}{a}\right)\right. \\
& \left.\times \exp \left[-\frac{c^{2}}{\gamma^{2}}(a-\operatorname{sgn} \xi)|\xi|\right]\right\} .
\end{aligned}
$$

When $\xi$ is replaced by $t+x / c$ in Eq. A28, and when the result is combined with Eqs. A14, A16, and A19, we have the results Eqs. 51, 53 of Sec. III.

A2 G. N. Watson, Theory of Bessel Functions (Cambridge University Press, Cambridge, England, 1958), pp. 186-187 and p. 80. 